\section{0-385 THE IMPACT OF WORKPLACE SOCIAL SUPPORT ON MENTAL HEALTH FUNCTIONING: A COHORT STUDY OF THE WORKFORCE IN STOCKHOLM COUNTY, SWEDEN}

${ }^{1}$ Kathryn Badarin, Andreas Lundin, Yvonne Forsell. ${ }^{1}$ Karolinska Institutet, Sweden

10.1136/OEM-2021-EPI.111

Introduction The burden of mental illness is pervasive. Improved methods for early detection of mental ill-health and comprehensive understandings of factors associated with declining mental health are needed. Workplace social support is an important factor for addressing employee's mental health. However, only a few studies have explored how workplace social support impacts on mental health from a functional perspective.

Objectives To investigate the prevalence of limited Mental Health Functioning (MHF) among Swedish workers and the association between workplace social support and MHF.

Methods This cohort study used a sample of 4979 employees from Stockholm County who responded to population-based questionnaires in 2000 and 2002. Two subsamples were explored: workers without baseline limitations in MHF $(\mathrm{n}=2844)$ and workers with baseline limitations in MHF $(n=2135)$. Exposure to workplace social support was estimated using the Swedish Demand-Control-Support Questionnaire. Follow-up data on MHF were taken from the World Health Organisation's Brief Disability Questionnaire. Multinomial regression analysis generated odds ratios (OR) with 95\% confidence intervals (CI95\%) adjusting for age, sex, education, socio-economic classification, cohabitation and social support outside the workplace.

Results In total, 43\% (37\% moderate and 6\% severe) of workers had limited MHF at baseline. A higher level of workplace social support was associated with a reduced likelihood of moderate functional limitations (AOR 0.92 95\%CI, 0.890.95 ) or severe functional limitations (AOR 0.87 95\%CI, $0.80,0.94)$ two years later. Among workers with pre-existing functional limitations, increased workplace social support was associated with improved MHF at follow-up (AOR 1.08 95\% CI, 1.05, 1.12).

Conclusions Limited MHF was prevalent among workers. We observed a protective relationship between increased workplace social support and limited MHF. Increased social support was also associated with improved MHF among workers with limited MHF. Interventions that increase workplace social support might protect against a deterioration in MHF and improve MHF among workers with preexisting limitations.

\section{Psychosocial-2}

\section{0-26 WORK-LIFE INTERFERENCE AND SUBSEQUENT LONG- TERM SICKNESS ABSENCE. A LONGITUDINAL STUDY OF THE SWEDISH WORKING POPULATION.}

${ }^{1}$ Emma Hagqvist, Ulrik Lidwall, Constanze Leineweber. 'Stockholm University, Sweden

\subsection{6/OEM-2021-EPI.112}

Introduction The literature consistently shows that work-life interference, i.e. the multiple burden of having different social roles, e.g. being a worker, parent, partner and friend is associated with poor health. However, few studies have studied the relationship between measures of work-life interference and objective measures of sickness absence.

Objectives The aim of this study is to investigate work-life interference as a risk factor for subsequent long-term sickness absence.

Methods Data were derived from four (2010-2016) waves of the Swedish Longitudinal Occupational Survey of Health. Data were linked to register data on long-term sickness absence (having at least one continuous period of medically certified sick leave exceeding 14 days) from the Swedish social insurance agency. Generalized estimating equations (GEE) with odds ratios (OR) and confidence interval of 95\% was used. The sample included 15244 individuals $(43.1 \%$ men and $56.9 \%$ women).

Results Almost one fifth of the sample, $18.7 \%$ (1110) started at least one period of long-term sickness absence at any point between first wave 2010 and two years after the last wave up to year 2018. Of those $1100,43.9 \%$ were men and $56.1 \%$ were women. Results show that work-life interference is associated with an increased risk for subsequent long-term sickness absence (OR: 1.55; 95\% CI=1.44-1.67) even when adjusting for general health $(\mathrm{OR}=1.39 ; 95 \% \mathrm{CI}=1.29-1.51)$ and other relevant factors (gender, having children living at home, having a partner, socioeconomic position, management position and age). We found no significant moderating effect of gender nor of time.

Conclusion We conclude that work-life interference is a risk factor for subsequent long-term sickness absence for working men and women in Sweden. From a public health perspective, improving possibilities to balance demands between work and private life can reduce risks of long-term sickness absence.

\section{0-56 WORKPLACE VIOLENCE AMONG WOMEN IN INDUSTRIAL FACTORIES}

'Alireza Dehdashti. 'Medical Sciences University of Semnan, Islamic Republic of Iran

\subsection{6/OEM-2021-EPI.113}

Introduction Workplace violence concerns a form of aggression encompassing behaviors that are intended to cause physical and mental harm. While studies reported violence is a major and increasing concern for the work environment in all countries, violence against female workers in the industrial sector has received less attention.

Objectives This study investigated the prevalence of various types of violence and associated factors among female workers in the industrial sector.

Methods This descriptive survey was conducted in eleven industrial sites in Iran. Cross-sectional data were collected by a 78-item questionnaire from 817 female workers recruited through workplace safety and wellbeing units inside the factories. The study measured three types of violence involving physical attacks, verbal threats, and sexual aggressions. Multiple logistic regression analyses were used to analyze how demographic characteristics and occupational factors were associated with women's perceived violence in work life.

Results About half of the participants reported having experienced at least one type of violence. The prevalence rates of verbal aggression, sexual harassment, and physical violence were $52.5 \%, 12 \%$ and $11.5 \%$, respectively. Single female workers (adjusted odds ratio $[\mathrm{AOR}]=2.06,95 \%$ $\mathrm{CI}=1.09-4.27)$, and age older than 35 years were 